\title{
Evidence for a modality effect in sentence retention
}

\author{
RALF RUMMER and JUDITH SCHWEPPE \\ Saarland University, Saarbrücken, Germany
}

\begin{abstract}
It is well known that an acoustic-sensory code supports retention of linguistic materials whose storage is particularly based on phonological information (e.g., unrelated word lists). The present study investigates whether such a code also contributes to the retention of sentences. It has been shown that short-term sentence recall particularly depends on propositional and lexicosemantic information, which are assumed to be supplied independently of modality influences. We employed the intrusion paradigm of Potter and Lombardi (1990) and manipulated the availability of acoustic-sensory information. Participants were instructed to read sentences either silently or aloud. Since these two reading conditions also differ with respect to articulatory information, a further condition that provided articulatory but not acoustic-sensory information was introduced (i.e., silent mouthing). Our data suggest that acoustic-sensory information is used, if available, even in sentence recall.
\end{abstract}

Short-term memory for unrelated word lists differs in many respects from memory for syntactically structured linguistic material. Although short-term list retention depends particularly on phonological information (see Baddeley, 1986), memory for sentences is mainly based on conceptual-propositional and lexicosemantic representations (see, e.g., Potter \& Lombardi, 1990). Conceptualpropositional information refers to semantic representations of the content of sentences, whereas lexicosemantic information concerns the meanings of the words included in a sentence. Recently, the experimental paradigms originally developed to demonstrate the importance of conceptual-propositional and lexicosemantic information have been used to investigate modality effects in sentence retention. These studies have demonstrated better immediate recall for auditory sentence presentation than for visual sentence presentation (Park \& Martin, 2002; Rummer \& Engelkamp, 2001). In these experiments, visual presentation was based on rapid serial visual presentation (RSVP). Because it is plausible to assume that RSVP leads to weaker phonological representations than do auditory and slow visual presentation (see Potter, 1999), this modality effect can be explained in two ways: The advantage for auditory presentation can be explained in terms of better phonological encoding and/or the contribution of acoustic-sensory information.

This research was supported by Grant Ru891/1-2 from the Deutsche Forschungsgemeinschaft (German Research Foundation). We are grateful to Ralph Dirksen, Jasmin Prokasky, and Jennifer Winnen, who performed the experiment and helped analyze the data. For valuable comments on an earlier version of this article, we thank Nelson Cowan, James Nairne, Mary Potter, Christoph Scheepers, and an anonymous reviewer. Correspondence relating to this article may be sent to R. Rummer, Saarland University, Department of Psychology, P.O. Box 151 150, 66041 Saarbrücken, Germany (e-mail: r.rummer@rz.uni-sb.de).
The use of phonological information in immediate sentence recall has been demonstrated both in neuropsychological patient studies (see, e.g., Martin, Shelton, \& Yaffee, 1994) and in experiments with unimpaired participants (e.g., Park \& Martin, 2002). Whether acoustic-sensory information also contributes to sentence repetition has been addressed in only a few studies (e.g., Balota, Cowan, \& Engle, 1990; Rummer \& Engelkamp, 2003), and the interpretation of most of these studies is ambiguous.

The term acoustic-sensory information refers to sensory representations of sounds and is not restricted to linguistic information. An acoustic code is extremely sensitive to acoustic interference (see, e.g., Conrad \& Hull, 1968; Cowan, 1995; Crowder, 1967; Penney, 1989). It is provided through auditory presentation only. The modality effect has been observed with both written and oral recall. However, there is some evidence that it is stronger with written than with oral recall (e.g., Penney, 1979).

A common test of the modality effect contrasts silent reading and reading aloud of unrelated lists of words, nonwords, letters, or digits (see, e.g., Conrad \& Hull, 1968; Frankish, 1985). It is assumed that both silent reading and reading aloud provide phonological information and that only reading aloud provides acoustic-sensory information. This additional information results in better recall performance, particularly for the most recent items. Unfortunately, reading aloud does not only differ from silent reading in providing an additional acoustic code. In contrast to silent reading, it also provides articulatory information. To dissociate these two possible sources of the modality effect, a condition that provides articulatory but not acoustic-sensory information has to be introduced. In this condition, participants are instructed to silently mouth the list items during encoding (e.g., Cheng, 1974). Hence, the modality effect would only reflect the use of acousticsensory information if there was a significant advantage 
for reading aloud over both mouthing and silent reading (but see Nairne \& Walters, 1983).

Although the contribution of acoustic information to list retention has repeatedly been demonstrated (a recent example is Cowan, Saults, \& Brown, 2004), it is less clear whether such information is used in sentence recall as well. First evidence for this assumption stems from Balota et al. (1990) and Rummer and Engelkamp (2003). The present study aims at demonstrating that the modality effect is not restricted to those materials whose short-term serial recall is mainly based on phonological information (e.g., unrelated lists of words, digits, or letters) but can also be found in sentence recall, which is plausibly assumed to be based on conceptual information (Potter \& Lombardi, 1990).

In the following section, we briefly describe an experimental paradigm introduced to demonstrate the dominance of conceptual (i.e., propositional and lexicosemantic) information in sentence regeneration. Subsequently, we will explain how this paradigm can be applied to test the critical question of this article.

\section{The Potter-Lombardi Intrusion Paradigm}

Potter and Lombardi (1990) stated that a "sentence is generated in immediate recall from a representation of its meaning using recently activated words" (p. 633). To test this conceptual regeneration hypothesis (i.e., the dominance of propositional-conceptual information and the additional use of lexicosemantic information), in each trial participants were presented with a list of semantically unrelated words and a sentence. Sentences and word lists were presented using RSVP. Participants' main task was to recall the presented sentence as accurately as possible. To make sure that the words of the unrelated word list were actively processed, participants were presented as well with a probe word, and they then had to decide whether the probe word appeared in the word list or not. The critical experimental manipulation concerned the words presented within the word lists: Half of the word lists included a word (termed the lure word) with a meaning similar to the target word in the sentence, and the other half included a word (termed the control word) that was conceptually unrelated to the target word. The sentences were composed in such a way that the participants tended to select the lure word instead of the target word during recall. Figure 1 illustrates the trial structure of their experiment.

Potter and Lombardi (1990) distinguished two kinds of lure intrusions: First, there was a high proportion of spontaneous lure intrusions; that is, even when the lure word was not included in the word list, participants tended to replace the target word with a lure word that the authors assumed fit the sentence context better than did the target word. Second, the lure word replaced the target word more often if the lure was included in the list than if the control word was included (induced lure intrusions). That is, participants produced sentences like "The knight rode around the castle searching for a place to enter" (when the actual target word was palace) more often if the list included the word castle. If there are more lure intrusions when the list includes a lure word, we speak of a lure intrusion effect. Because lure words and target words differed with respect to their phonological surfaces, these results were taken as evidence for the conceptual regeneration hypothesis. The same paradigm can also be used to test the contribution of other types of information. If, for instance, the provision of phonological information decreases or prevents the lure intrusion effect, this can be taken as evidence for the contribution of such information to sentence recall.

\section{Testing the Contribution of Acoustic-Sensory Information to Sentence Retention}

In order to apply the intrusion paradigm to the topic of this article, the paradigm has to be modified as follows: First, sentences have to be presented using simultaneous visual presentation instead of RSVP. Second, presentation times have to be long enough to enable reading at a

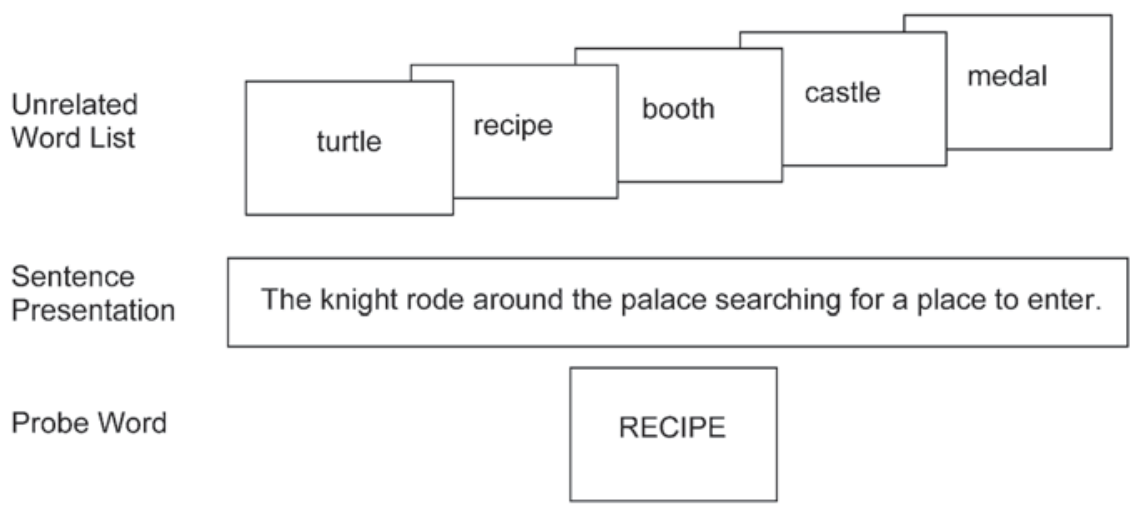

Sentence Recall

"The knight rode around the castle searching for a place to enter."

Figure 1. Trial structure of the intrusion paradigm in Potter and Lombardi (1990, Experiment 2). Our design differed because the target word was always one of the final three words of the sentence. 
normal rate. ${ }^{1}$ Third, since acoustic-sensory information is only available for the most recent part of the sentence, the target noun has to be one of the three most recent words. Fourth, a condition in which the sentences have to be read aloud and a condition in which they have to be mouthed silently should be added to the silent reading condition. In all of these conditions, the preceding word lists should be read silently. To demonstrate that there is a benefit of the acoustic code derived from reading aloud per se, a further presentation condition, in which both the word lists and the sentences have to be read aloud, should be introduced. If an acoustic advantage protecting against lure intrusions occurred even with read-aloud lists, one could assume that it was not the difference between list and sentence modalities, and thus the easier discrimination of list and sentence sources, that provided this protection.

For those conditions providing acoustic-sensory information (i.e., the two reading-aloud conditions), we expected no lure intrusion effect (i.e., no difference between spontaneous and induced lure intrusions), whereas for those conditions lacking acoustic-sensory representations (i.e., silent reading and mouthing), a lure intrusion effect should be observed. In addition, we expected no difference between the two reading-aloud conditions on one hand and between the mouthing and silent-reading conditions on the other.

\section{The Experiment}

The present experiment was based on a $2 \times 4$ design with the within-subjects factor lure condition (list with a semantically related lure word or an unrelated control word) and the between-subjects factor reading condition (silent reading, mouthing, reading aloud 1 , or reading aloud 2). Reading aloud 1 refers to the condition in which only the target sentence was read aloud, whereas reading aloud 2 stands for the condition in which both sentence and word list were vocalized.

\section{METHOD}

\section{Participants}

Sixty-four students from Saarland University participated in the experiment. All of them were native speakers of German. A quarter of the individuals (i.e., 16) were randomly assigned to each of the four experimental groups.

\footnotetext{
Materials

The participants were presented with 20 sentences and 20 word lists. Each sentence included a target word, which was one of the three most recently encountered words in the sentence. In 10 of these sentences, the target word was the last word; in 7 cases, it was the second to last; and in only 3 cases, the target noun was followed by two further words. (A complete list of the materials can be found at www.uni-saarland.de/fak $5 /$ wintermantel/hp/ralf/mod-effect.htm.) The word lists consisted of five unrelated nouns, which included a lure word in 10 lists and a control word in the remaining 10. In the lure condition, the lists included a word that was semantically similar to the target word. These lure words were chosen in such a way that they appeared more suitable in the context of the sentence than did the target word itself. In the control condition, a noun that was neither semantically nor phonologically related to the target word
}

replaced the lure word. Except for the lure word/control word, the two word lists were identical.

\section{Procedure}

Half of the sentences were presented with a list that included a lure word, and the other half were presented with a list that included a control word. Across participants, each sentence appeared equally often with a list containing a lure word or a control word. The assignment of the lists to one of these conditions was determined at random, as was the presentation order of the 20 sentence-list combinations. In all trials, the list preceded the sentence. The structure of a single trial was as follows: The participants started a trial by pressing the space bar of the computer keyboard. Three asterisks appeared for $300 \mathrm{msec}$ in the center of the screen, followed by a blank screen for $350 \mathrm{msec}$. Subsequently, the five nouns of the word list were presented, one at a time, for $250 \mathrm{msec}$ per word. In all but one experimental condition, the lists had to be read silently. The sentence was presented after an interval of $250 \mathrm{msec}$, during which a row of percent signs was displayed. The words of each sentence were presented simultaneously as one string of words on the computer screen (presentation time was 4,462 msec per sentence, corresponding to an average of $350 \mathrm{msec}$ per word).

The first group of participants had to read the sentences silently; a second group had to mouth them silently while reading; a third group read the sentences aloud and the lists silently; and the last group vocalized both the lists and the sentences. The mouthing group was instructed to move their lips and tongues as though they were speaking but without voicing the sentence. As soon as the sentence had vanished from the screen, a row of percent signs appeared for $517 \mathrm{msec}$. Finally, the probe word was presented in capital letters for $500 \mathrm{msec}$. The participants had to decide whether or not it had been part of the word list, and they responded by pressing one of two response keys (the right or the left shift key for yes or no, respectively). They were told that this matching task served to increase the difficulty of oral sentence recall, which had to be done immediately thereafter. Before the experiment started, six practice trials designed like the experimental trials were presented to the participants.

The sentences were recorded and transcribed. The overall recall accuracy (excluding the target word) and the proportion of omissions and nonlure substitutions of the target word were taken as control variables. The critical dependent variable of the experiment was the percentage of lure intrusions for the target word.

\section{RESULTS}

\section{Recall Accuracy}

As an overall measure of recall performance, we report the percentage of words correctly reproduced in the correct order. The target word was excluded from analysis. The statistical analyses were based on a one-way ANOVA, including the conditions silent reading, mouthing, reading aloud 1 , and reading aloud 2 .

Figure 2 includes the means and standard errors for general recall performance as a function of reading modality.

The ANOVA did not reveal a significant effect for the factor reading modality $[F(3,60)=1.46, p>.1]$.

\section{Omissions and Substitutions of the Target Word}

To test whether or not the four reading conditions differed with respect to the encoding accuracy of the target word, we recorded cases in which the target word was omitted or replaced by other nouns but not by the lure word. One-way ANOVAs did not reveal a significant effect $\left[F_{1}(3,60)=1.43, p>.2 ; F_{2}(3,57)=2.51, p>.05\right]$, and neither did least significant difference (LSD) tests. 


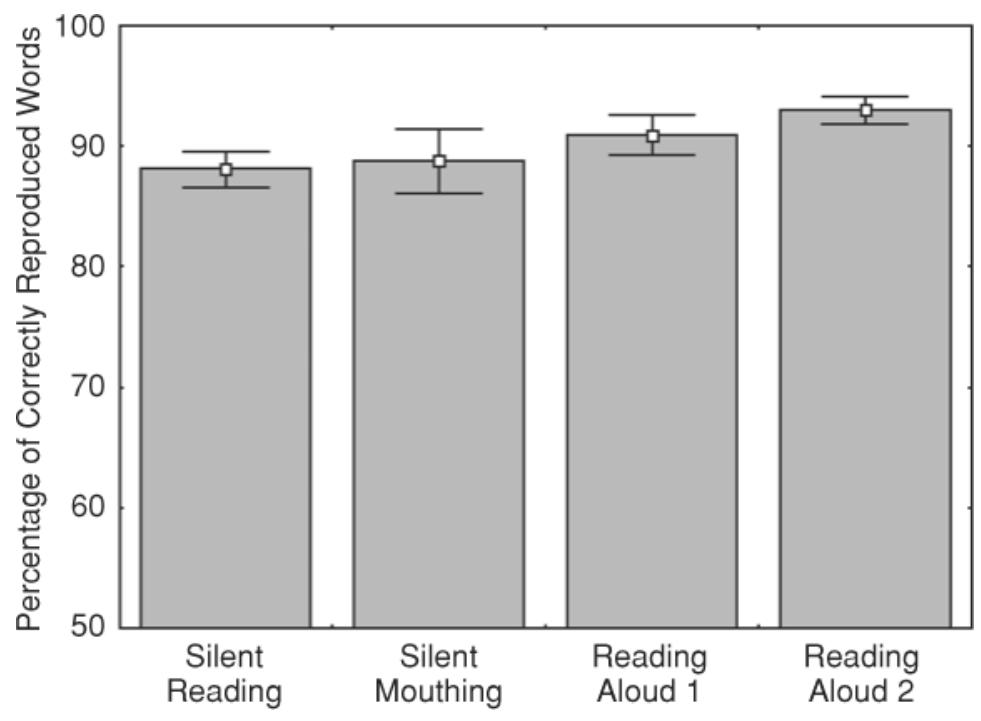

Figure 2. Means and standard errors of correctly reproduced words per sentence (excluding the target noun) as a function of reading condition (i.e., silent reading, silent mouthing, reading aloud 1 , and reading aloud 2).

\section{Lure Intrusions}

Figure 3 depicts the percentage of lure intrusions as a function of the two independent variables, reading condition and lure condition.

For the present study, we assumed that the intrusion effect would be modified by whether or not acoustic-sensory information was provided during sentence presentation.
A two-way interaction between the lure factor and whether or not acoustic-sensory information was provided was critical to sustain this hypothesis. The statistical analyses followed a two-step procedure: First, we conducted separate analyses for the two reading-aloud groups (i.e., reading aloud 1 and reading aloud 2) and for the two silent groups (i.e., silent reading and mouthing). For both analyses, we

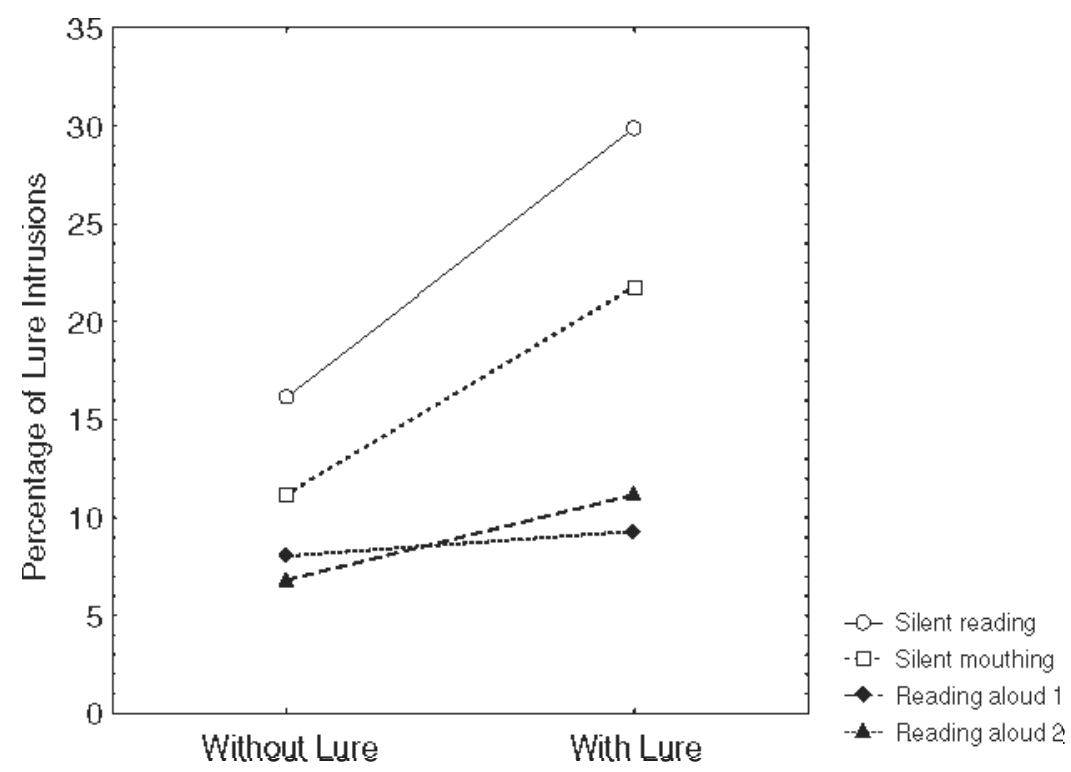

Figure 3. Mean percentages of lure intrusions as a function of reading condition (i.e., silent reading, silent mouthing, reading aloud 1, and reading aloud 2) and lure condition (i.e., list with a control word vs. list with a lure word). Those conditions in which an acoustic-sensory code for the target word was available are marked with filled symbols; those in which acoustic-sensory information was not available are represented by unfilled symbols. 
expected that the two-way interactions between reading condition and lure factor would not reach significance. We further expected that the lure factor would not reach significance for conditions in which acoustic-sensory information was provided; in contrast, conditions in which acoustic-sensory information was not provided should reveal a significant main effect for the lure factor.

Second, planned comparisons were calculated that collapsed the two reading-aloud conditions as well as the silent-reading and mouthing conditions. Here, we expected a significant two-way interaction between the collapsed factor acoustic-sensory information (acoustic code provided vs. not provided) and the lure condition (without vs. with lure).

The separate ANOVAs that compared the two groups in which acoustic-sensory information was provided did not reveal a significant two-way interaction between reading condition (reading aloud 1 and 2) and lure condition (without vs. with lure) $\left(F_{1}\right.$ and $\left.F_{2}<1\right)$. As expected, the main effect for lure condition did not reach significance, either $\left[F_{1}(1,30)=1.91, p>.1 ; F_{2}(1,19)=1.21, p>.1\right]$, nor did the main effect for reading condition $\left(F_{1}\right.$ and $\left.F_{2}<1\right)$.

The separate ANOVAs comparing the two groups in which no acoustic-sensory information was provided did not reveal a significant interaction, either $\left(F_{1}\right.$ and $\left.F_{2}<1\right)$. However, as expected, a significant main effect for lure condition was found $\left[F_{1}(1,30)=14.75, p<.01\right.$; $\left.F_{2}(1,19)=15.22, p<.001\right]$. The main effect for reading factor (silent reading vs. mouthing) did not reach significance $\left[F_{1}(1,30)=3.47, p>.05 ; F_{2}(1,19)=2.88\right.$, $p>.1]$.

The analyses collapsed across the two reading-aloud conditions on one hand and silent reading and mouthing on the other revealed the expected significant two-way interaction between the acoustic-sensory information and lure factors $\left[F_{1}(1,60)=6.19, p<.025 ; F_{2}(1,19)=6.89\right.$, $p<.025] . F$ ratios for this interaction were even higher if the analyses were restricted to those 10 items in which the target noun was the most recently encountered word of the sentence $\left[F_{1}(1,60)=8.66, p<.01 ; F_{2}(1,9)=7.81, p<\right.$ $.025]$. If acoustic-sensory information was provided, $7 \%$ spontaneous and $10 \%$ induced intrusions were found; if no acoustic-sensory information was provided, we observed $14 \%$ spontaneous and $26 \%$ induced intrusions. LSD tests revealed that the difference between spontaneous and induced intrusions was only significant in the nonacoustic condition.

\section{DISCUSSION}

The critical question we asked in the present study was whether or not acoustic-sensory information would be used in sentence recall. Since the acoustic code should be useful to distinguish between target and lure words, it should help prevent lure words (i.e., words that are semantically but not phonologically related to the target noun of a sentence) from intruding on sentence recall. To vary the availability of an acoustic code, we had our participants read sentences under four conditions: The first group had to read the sentences silently, the second mouthed the sentences silently while reading, the third read the sentences aloud and the lists silently, and the fourth vocalized both the word lists and the sentences during reading. Acoustic-sensory information was available only to the two reading-aloud conditions. We expected that those conditions that were identical with respect to the provision of acoustic-sensory information should not differ from each other. The separate ANOVAs supported this assumption.

The proportion of lure intrusions did not vary in the two reading-aloud conditions: In either one, a lure intrusion effect was observed. Here, the most important finding is that the lure intrusion effect was not influenced by whether or not the preceding word list was read in the same modality as the sentence. Thus, the absence of an intrusion effect cannot be attributed to an advantage in distinguishing the target from the lure source. Hence, it is the acoustic-sensory representation of the target word that helps to prevent lure intrusions.

The two conditions without acoustic-sensory information (i.e., silent reading and mouthing) did not differ from each other, either. However, for these conditions, we also observed the expected lure intrusion effect: That is, we found a higher proportion of lure intrusions when the lure word was present in the word list than when it was absent (control condition). This finding suggests that neither phonological information alone nor phonological plus articulatory information is sufficient to prevent a lure intrusion effect. In addition, this finding rules out any explanation in which the modality effect is attributed to articulatory rather than acoustic-sensory information.

Our basic hypothesis concerning the difference between those conditions that provide an acoustic code and those that do not was directly tested using planned comparisons. This analysis revealed a significant two-way interaction between the collapsed factor acoustic-sensory information (present or absent) and the lure factor (list with or without lure word). If acoustic-sensory information was available during sentence recall, no lure intrusion effect was observed; in contrast, if no acoustic-sensory code was available, induced intrusions occurred more often than spontaneous intrusions.

An acoustic code is extremely sensitive to interference caused by subsequent auditory stimuli. Therefore, modality effects can be demonstrated for the last one, two, or three items of a list only. Some data suggest that the contribution to list recall is restricted to the very last item (see, e.g., Nairne \& Walters, 1983); others suggest that the acoustic-sensory code contributes to the last three items (e.g., Frankish, 1985). In any case, it is plausible to assume the strongest modality effect for the most recent item. In order to test this assumption, we conducted analyses including only those sentences in which the target noun was the final word. Given the reduced power of these analyses (only half of the sentences were included), the observation of a critical two-way interaction between the collapsed reading-aloud conditions on one hand and the silent-reading and silent-mouthing conditions on the 
other is further support for the hypothesis that the modality effect observed here is indeed due to the contribution of acoustic-sensory information.

A possible objection against this interpretation is that reading aloud simply improves overall comprehension of the sentence (or target), which in turn lowers overall intrusion rates. We do not think that this interpretation is appropriate for two reasons: First, there is no empirical evidence that reading aloud leads to better comprehension than does silent reading (see, e.g., Narvaez, van den Broek, \& Barrón Ruiz, 1999), and second, comprehension was close to perfect in all four reading conditions. A reanalysis of the recalled sentences showed that the participants did not produce any errors that changed the content of the sentences.

This second point raises another question: In this experiment, why does the availability of acoustic-sensory information influence the proportion of lure intrusions but not the accuracy of general recall? The sentences were designed in such a way that the most recently read part could be easily predicted from the beginning and middle of the particular sentence. The only exception was the target word, for which the context allowed or even encouraged replacement by the lure word. Hence, with respect to the region of the sentence for which acoustic-sensory information was potentially available, the target noun was the only word for which the measurement of acoustic-sensory involvement was possible.

In sum, the present article offers a detailed investigation concerning the source of the modality effect in sentence retention. Until now, this effect has particularly been investigated for short-term retention of unrelated lists of words, nonwords, digits, and letters, and the retention of all of these stimuli is based mainly on phonological shortterm memory (for an overview, see, e.g., Baddeley, 1986). The present study demonstrates that the modality effect also occurs with sentence regeneration, an experimental task that is primarily based on conceptual information and in which surface representations (e.g., phonological information) play only a minor role. Yet even under these circumstances, a modality effect can be demonstrated. Furthermore, the modality effects observed in sentence regeneration and in list recall reflect the same mechanisms, and both are due to the contribution of acoustic-sensory codes.

\section{REFERENCES}

BADDELEy, A. D. (1986). Working memory. Oxford: Oxford University Press.
Balota, D. A., Cowan, N., \& Engle, R. W. (1990). Suffix interference in the recall of linguistically coherent speech. Journal of Experimental Psychology: Learning, Memory, \& Cognition, 16, 446-456.

Cheng, C.-M. (1974). Different roles of acoustic and articulatory information in short-term memory. Journal of Experimental Psychology, 103, 614-618.

Conrad, R., \& Hull, A. J. (1968). Input modality and the serial position curve in short-term memory. Psychonomic Science, 10, 135-136.

Cowan, N. (1995). Attention and memory: An integrated framework. New York: Oxford University Press.

Cowan, N., Saults, J. S., \& Brown, G. D. A. (2004). On the auditory modality superiority effect in serial recall: Separating input and output factors. Journal of Experimental Psychology: Learning, Memory, \& Cognition, 30, 639-644.

Crowder, R. G. (1967). Prefix effect in immediate memory. Canadian Journal of Psychology, 21, 450-461.

FRANKISH, C. (1985). Modality-specific grouping effects in short-term memory. Journal of Memory \& Language, 24, 200-209.

Martin, R. C., Shelton, J. R., \& Yaffee, L. S. (1994). Language processing and working memory: Neuropsychological evidence for separate phonological and semantic capacities. Journal of Memory \& Language, 33, 83-111.

NAIRne, J. S., \& Walters, V. L. (1983). Silent mouthing produces modality- and suffix-like effects. Journal of Verbal Learning \& Verbal Behavior, 22, 475-483.

Narvaez, D., van den Broek, P., \& Barrón Ruiz, A. (1999). The influence of reading purpose on inference generation and comprehension in reading. Journal of Educational Psychology, 91, 488-496.

PARK, N., \& Martin, R. C. (2002, March). A contribution of phonological representations to immediate sentence recall. Poster presented at the 15th Annual CUNY Conference on Human Sentence Processing, New York City.

Penney, C. G. (1979). Interactions of suffix effects with suffix delay and recall modality in serial recall. Journal of Experimental Psychology: Human Learning \& Memory, 5, 507-521.

PenNey, C. G. (1989). Modality effects in the structure of short-term verbal memory. Memory \& Cognition, 17, 398-422.

PotTer, M. C. (1999). Understanding sentences and scenes: The role of conceptual short-term memory. In V. Coltheart (Ed.), Fleeting memories: Cognition of brief visual stimuli (pp. 13-46). Cambridge, MA: MIT Press.

PotTer, M. C., \& Lombardi, L. (1990). Regeneration in the short-term recall of sentences. Journal of Memory \& Language, 29, 633-654.

Rummer, R., \& Engelkamp, J. (2001). Phonological information contributes to short-term recall of auditorily presented sentences. Journal of Memory \& Language, 45, 451-467.

Rummer, R., \& EngelKamp, J. (2003). Short-term sentence recall: Evidence for the contribution of acoustic-sensory information. Acta Psychologica, 114, 67-82.

\section{NOTE}

1. Recently, Rummer and Engelkamp (2003) compared silent reading and reading aloud of simultaneously but rapidly presented sentences, and they did not find a lure intrusion effect at all. From our point of view, the absence of this effect in their experiment, even with silent reading, was due to incomplete phonological recoding in those conditions.

(Manuscript received March 9, 2004; revision accepted for publication March 15, 2005.) 